\title{
On the Particle Scattering Factor of Janus Micelles
}

\author{
Tobias Fütterer ${ }^{a}$, Gerrit A. Vliegenthart ${ }^{b}$, Peter R. Lang $^{b *}$ \\ ${ }^{a}$ Iwan Stranski Laboratorium, TU-Berlin, Strasse des 17 Juni 112, 10623 Berlin, Germany \\ ${ }^{\mathrm{b}}$ Forschungszentrum Jülich, IFF, 52425 Jülich, Germany \\ Corresponding author’s e-mail address: p.lang@fz-juelich.de \\ RECEIVED DATE (to be automatically inserted after your manuscript is accepted if \\ required according to the journal that you are submitting your paper to)
}

\begin{abstract}
We calculate scattering curves from Janus micelles based on a new particle scattering factor which we tested against Monte-Carlo simulations. If we compare scattering data from Janus micelles with data of core shell particles, we find that it is virtually impossible to distinguish the particle scattering factors of the two structures, if the scattering length densities of the respective segments are similar. As intuitively expected this situation worsens if the particles have a finite spread of radii and/or scattering length densities. We show however, that even in this case a positive identification of Janus-micelles is possible on the basis of scattering experiments, if contrast variation techniques are applied. For this purpose the outer radius of the micelle should be larger than twice the inner radius, and the scattering length densities of the two Janus face
\end{abstract}


segments should differ at least by a factor of two. The most favorable conditions occur when the scattering length density of the core is the mean value of the two outer segments.

INTRODUCTION During the last decade polymer chemist have taken a great effort to synthesize so-called Janus-micelles, a term which was coined, referring to the ancient two faced roman deity. Accordingly Janus-micelles are self assembled structures, in which the surface area of the aggregate is micro-phase separated into two "faces". A general route to the synthesis of these structures is the use of two blockcopolymers A-B and A-C, which are dissolved in a medium that is a solvent for the B- and the C-block but a non-solvent for the A-block. The selectivity of the solvent will lead to the formation of micelles in the first place, in which the insoluble A-blocks are separated from the continuous medium by the soluble blocks. However, as polymers are generally chemically incompatible, it may be expected that the C- and the B- blocks separate to form nanometer scaled domains of either block.

Typically Janus-particles have spherical shape ${ }^{1}$, although there are reports on nonspherical particles with Janus-type internal configuration ${ }^{2,3}$. In the present contribution we will limit the discussion to spherical particles because these are of particular interest for two reasons. First, they appear to be most easily synthesized. Second, the compartmentalization of material will as a consequence lead to symmetry break of the pair interaction potential, between two spherical particles. That is, Janus micelles are a convenient route to synthesize dipolar spheres, a type of structure which is being intensively investigated in colloidal physics ${ }^{4,5}$. The combination of spherical shape and non-spherosymmetric interaction potential leads to novel structures and phases not observed before ${ }^{6}$. Besides this fundamental aspects it is also expected that Janus micelles may have the potential for new applications in various fields. 
Experimental evidence for the internal structure of Janus-particles has been gathered from a variety of techniques (see e.g. ref 1), however, a sound proof of a Janus type compartmentalization is difficult to achieve for various reasons. Using modern imaging techniques like SFM or STEM the distinction between two faces of a micelle is very difficult due to lack of contrast. Structural analysis by scattering techniques is hampered by the fact, that Janus particles tend to aggregate into super structures. Furthermore there was no particle scattering function available for Janus particles until recently ${ }^{7}$. Even with a particle scattering factor available, it is not straightforward to identify Janus micelles in a scattering experiment because the scattering from Janus particles is merely indistinguishable from the scattering of centrosymmetric core shell particles, if the scattering contrast is not carefully selected. This is shown in Figure 2 and will be discussed in detail in the 'Discussion' section.

In the present contribution we will introduce a new particle scattering factor for Janus particles which we tested against Monte-Carlo calculations of the scattering function. In addition we describe a two step scheme of small-angle neutron scattering (SANS) data analysis which eventually allows a positive identification of Janus particles and the determination of structural parameters. The paper is organized as follows: in the section titled 'Particle scattering factor' we will discuss different competing structures for Janus particles, introduce a new particle scattering factor and relate it to the expression given by Kaya'. In the 'Discussion' and 'Conclusions' sections we will outline a two step scheme to analyse neutron scattering data applying contrast variation. Finally the derivation of the particle scattering factor will be given in the appendix.

PARTICLE SCATTERING FACTOR If Janus micelles are synthesized from a mixture of A-B and A-C blockcopolymers (BCP) according to the general route sketched in the introduction, there are three limiting structures to which the BCP may self assemble in a medium which is a 
solvent for the B- ant the C-block and a non-solvent for the A-block. If the B-blocks separate completely from the C-blocks and two hemispherical caps are formed, the desired configuration is obtained. As the opposite limit one might imagine a structure where the two blocks in the corona are evenly distributed. In between these two limits there could as well occur intermediate structures, in which one of the blocks, say the C-block, forms a continuous background with speckles of the other block, say B. The third limiting case is the formation of two populations of micelles one consisting of $\mathrm{AB}$ core-shell particles and the other of AC core-shell particles. In what follows we will confine the discussion to the two structures mentioned first, i. e. we will use the term Janus particle for an entity consisting of a spherical core with the scattering length density (SLD) $\rho_{\mathrm{A}}$ and the radius $R_{\mathrm{i}}$ covered with two hemispherical caps with the respective scattering length densities $\rho_{\mathrm{B}}$ and $\rho_{\mathrm{C}}$. The entire assembly has the radius $R_{\mathrm{O}}$ (see Figure 1 left). The term core shell particle will be assigned to the type of particles which is sketched in the middle of Figure 1, where the SLD of the shell is constant $\rho_{B C}=\left(\rho_{B}+\rho_{C}\right) / 2$ throughout the shell. The radius and the SLD of the core as well as the outer radius of the particle are the same as for the Janus particle, i.e. $R_{\mathrm{i}}, \rho_{\mathrm{A}}$ and $R_{\mathrm{o}}$ respectively. On the right side of Figure 1 we have also sketched the simplified Janus structure for which Kaya has published the particle scattering factor $^{7}$, i. e. two half spheres with radius $R_{0}$ divided by a plane.

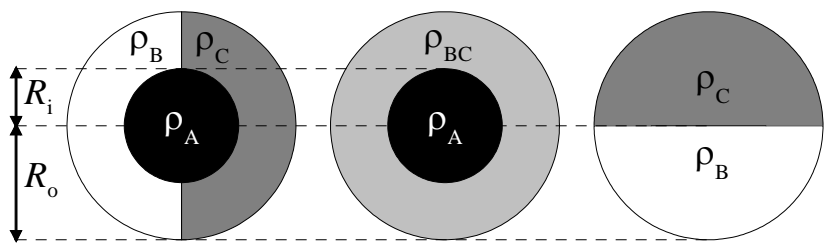

Figure 1 Sketch of competing particle structures. From left to right: Janus micelle, core shell particle and simplified Janus micelle. 
The particle scattering factors or form factors for objects which do not have spherical symmetry can be generally written as

$$
P(Q)=\frac{1}{4 \pi} \int_{0}^{2 \pi} d \varphi \int_{0}^{\pi} b(\mathbf{Q}, \theta)^{2} \sin \theta d \theta
$$

where the two integrals represent averaging of the particle orientation with respect to the scattering vector $\mathbf{Q}$. The form amplitude $b(\mathbf{Q}, \theta)$ is the normalized Fourier-transform of the excess SLD distribution in the particle with respect to the solvent.

$$
b(\mathbf{Q}, \theta)=\frac{\int \rho(\mathbf{r}) \exp \{i \mathbf{Q} \cdot \mathbf{r}\} d \mathbf{r}}{\int \rho(\mathbf{r}) d \mathbf{r}} .
$$

The integral in the denominator of eq. (2) is a normalization constant and will be called $V_{\mathrm{n}}$ in what follows. For a spherical particle with constant SLD it is the product of the sphere volume and the SLD.

For the Janus micelle (Figure 1 left), the square of the form factor amplitude is given by

$$
\begin{aligned}
b_{J}^{2}(\mathbf{Q}, \theta)=\frac{4 \pi^{2}}{Q^{2}\left(1-\mu^{2}\right) N_{n}^{2}} & \left(\left(\left(\rho_{B}+\rho_{C}\right) \int_{0}^{R_{o}} \cos (k z) F\left(R_{o}, z, \mu\right) d z\right.\right. \\
& \left.+\left(\Delta_{B A}+\Delta_{C A}\right) \int_{0}^{R_{i}} \cos (k z) F\left(R_{i}, z, \mu\right) d z\right)^{2} \\
& +\left(\left(\rho_{B}-\rho_{C}\right) \int_{0}^{R_{o}} \sin (k z) F\left(R_{o}, z, \mu\right) d z\right. \\
& \left.\left.+\left(\Delta_{B A}-\Delta_{C A}\right) \int_{0}^{R_{i}} \sin (k z) F\left(R_{i}, z, \mu\right) d z\right)^{2}\right)
\end{aligned}
$$


where $Q=|\mathbf{Q}|, \mu=\cos \theta, \mathrm{k}=Q \mu, \Delta_{B A}=\rho_{A}-\rho_{B}, \Delta_{C A}=\rho_{A}-\rho_{C}$ and the functions $F\left(R_{\mathrm{j}}, z, \mu\right)$ are given by $F\left(R_{j}, z, \mu\right)=\sqrt{R_{j}^{2}-z^{2}} J_{1}\left(Q \sqrt{1-\mu^{2}} \sqrt{R_{j}^{2}-z^{2}}\right)$, with $J_{1}$ the Bessel function of first order. For this structure the normalization constant is $V_{n}=\frac{2}{3} \pi\left(\left(\rho_{B}+\rho_{C}\right) \cdot\left(R_{o}^{3}-R_{i}^{3}\right)+2 \rho_{A} \cdot R_{i}^{3}\right)$.

For the two half sphere structure (Figure 1 right) the square of the form factor amplitude reduces to the expression given by Kaya ${ }^{7}$, which in our notation reads

$$
\begin{array}{r}
b_{h s}^{2}(\mathbf{Q}, \theta)=\frac{4 \pi^{2}}{Q^{2}\left(1-\mu^{2}\right) N_{n}^{2}}\left(\left(\left(\rho_{B}+\rho_{C}\right) \int_{0}^{R_{o}} \cos (k z) F\left(R_{o}, z, \mu\right) d z\right)^{2}\right. \\
\left.+\left(\left(\rho_{B}-\rho_{C}\right) \int_{0}^{R_{o}} \sin (k z) F\left(R_{o}, z, \mu\right) d z\right)^{2}\right)
\end{array}
$$

In this case the normalization constant reduces to $V_{n}=\frac{2}{3} \pi \cdot\left(\rho_{B}+\rho_{C}\right) \cdot R_{O}^{3}$

While the calculation of form factors using eq. (3) or eq. (4) has to be done numerically, the particle scattering factor of a core-shell structure (Figure 1 middle) can be written in a closed analytical form as ${ }^{8}$

$$
P_{C S}(Q)=\left(\frac{4 \pi}{Q^{3} V_{n}}\left[\left(\rho_{A}-\rho_{B C}\right)\left(\sin \left(Q R_{i}\right)-Q R_{i} \cos \left(Q R_{i}\right)\right)+\rho_{B C}\left(\sin \left(Q R_{O}\right)-Q R_{O} \cos \left(Q R_{O}\right)\right)\right]\right)^{2}
$$

where $V_{n}=\frac{4}{3} \pi \cdot\left(\rho_{A} R_{i}^{3}+\rho_{B C} R_{o}^{3}\right)$.

If particle interaction can be neglected, which we assume in what follows, the scattered intensity is related to the form factor by $I(Q)=V_{n}^{2} P(Q)$ for a single particle.

In the calculation of the SANS-curves presented in the next section we used normalized excess scattering length densities $\rho_{\mathrm{j}}$ which are related to absolute values as follows: 
$\rho_{j}=\frac{\rho_{j}^{a b s}-\rho_{D-\text { solvent }}^{a b s}}{\rho_{H-\text { solvent }}^{a b s}-\rho_{D-\text { solvent }}^{a b s}}$

for the particle segments and

$\rho_{\text {solvent }}=\frac{\rho_{\text {solvent }}^{a b s}-\rho_{D-\text { solvent }}^{a b s}}{\rho_{H-\text { solvent }}^{a b s}-\rho_{D-\text { solvent }}^{a b s}}$

for solvent mixtures. Here $\rho_{D-\text { Solvent }}^{a b s}$ and $\rho_{H-\text { Solvent }}^{a b s}$ are the absolute scattering length densities of a deuterated and a proteated solvent respectively. According to this convention, also calculated intensities will be normalized, and they are related to absolute values by

$$
I(Q)=\frac{I(Q)^{a b s}}{\left(\rho_{H-\text { solvent }}^{a b s}-\rho_{D-\text { solvent }}^{a b s}\right)^{2}}
$$

To mimic real SANS experiments as close as possible we took into account data smearing in the analytical calculation of the scattering curves. If not explicitly stated otherwise, the ideal model scattering functions were smeared with a wavelength distribution, an angular spread and a finite detector resolution applying a Gaussian-type resolution function as suggested by Barker et $\mathrm{al}^{9}$.

In order to calculate the scattered intensity using Monte Carlo simulations we consider a single spherical particle containing $\mathrm{N}$ scattering elements. The number density of scattering elements at a position $\mathbf{r}$ is $\eta(\mathbf{r})=\sum_{j}^{N} \delta\left(\mathbf{r}-\mathbf{r}_{j}\right)$ and the scattering length at this point is introduced through the weight function $w(\mathbf{r})$. Thus, the local scattering length density is $\rho(\mathbf{r})=\eta(\mathbf{r}) w(\mathbf{r})$.

The scattering intensity is given by,

$$
I(\mathbf{Q})=\langle\rho(\mathbf{Q}) \rho(-\mathbf{Q})\rangle
$$


where $\rho(\mathbf{Q})$ are the Fourier components of the scattering length density defined as $\rho(\mathbf{Q})=\int \rho(\mathbf{r}) \exp (i \mathbf{Q} \cdot \mathbf{r}) d \mathbf{r}=\sum_{j}^{N} w(\mathbf{r}) \exp (i \mathbf{Q} \cdot \mathbf{r})$ The form factor is defined as $\mathrm{P}(\mathbf{Q})=\mathrm{I}(\mathbf{Q}) / \mathrm{I}(\mathbf{Q}=0)$

In our simulations, we take $\eta(\mathbf{r})$ constant throughout the sphere and change the scattering properties in the sphere via the weight function $w(\mathbf{r})$. We put the center of the sphere at the origin and take the xy-plane as the dividing plane between the two outer half spheres. For each of the half spheres, $w(\mathbf{r})$ is only a function of the distance $r=|\mathbf{r}|$ to the center of the sphere. To model a smooth density profile from the core to the corona of the sphere we use a tanh-profile,

$$
w(r)=\left\{\begin{array}{l}
\frac{\rho_{B}-\rho_{A}}{2} \tanh \left(\frac{r-R_{i}}{d}\right)+\frac{\rho_{B}+\rho_{A}}{2} \text { for } z<0 \\
\frac{\rho_{C}-\rho_{A}}{2} \tanh \left(\frac{r-R_{i}}{d}\right)+\frac{\rho_{C}+\rho_{A}}{2} \text { for } z \geq 0
\end{array}\right.
$$

This functional form gives a gradual change of the scattering density between $\rho_{A}$ and $\rho_{B}$ or $\rho_{C}$ The parameter $d$ sets the sharpness of the interface between core and corona. The model does not take into account a diffuse interface between the two outer half spheres.

The actual MC calculations were performed as follows. An initial configuration was created by generating $N$ points randomly in a sphere of unit radius. To each of these $N$ points the appropriate weight $w(\mathbf{r})$ was attributed. Rotationally averaged intensities were determined by averaging $I(Q)$ over $M$ configurations obtained by rotating the sphere around a randomly chosen axis over a randomly chosen angle. For $N=100000$ and $M=100$ we find perfect agreement with analytical results.

DISCUSSION Usually SANS data are analyzed by non linear least squares fitting of the experimental data with an appropriate particle scattering factor. In Figure 2 the major problem of this procedure is illustrated. We have calculated form factors for a realistic system with the 
potential to form Janus micelle as it was synthesized by Erhardt et $\mathrm{al}^{1}$. According to there synthesis it is highly probable that the particles have a Janus type structure with a pulybutadiene core (A) and two half shells which consists of polystyrene blocks (B) and poly(methylmethacrylate) blocks (C) respectively. Based on the bulk densities of the three polymers and the deuterated solvent tetrahydrofuran the scattering length densities can be estimated to be $\rho_{A}=0.956, \rho_{B}=0.819$ and $\rho_{C}=0.808$. In the competing core shell structure in which the B- and C-blocks do not separate, the SLD of the shell is $\rho_{\mathrm{BC}}=0.813$. Using these SLDs we calculated the particle scattering factors of a Janus micelle and of the core shell particle which are shown in Figure 2. Here we did not take into account data smearing, nevertheless the curves are virtually identical. Even worse, in a real experiment tiny differences, if there were any, would be smeared due to the limited experimental resolution. It is thus obvious that a Janus micelle can not be distinguished from a core shell particle by one single scattering experiment, but elaborate contrast variation experiments have to be applied.

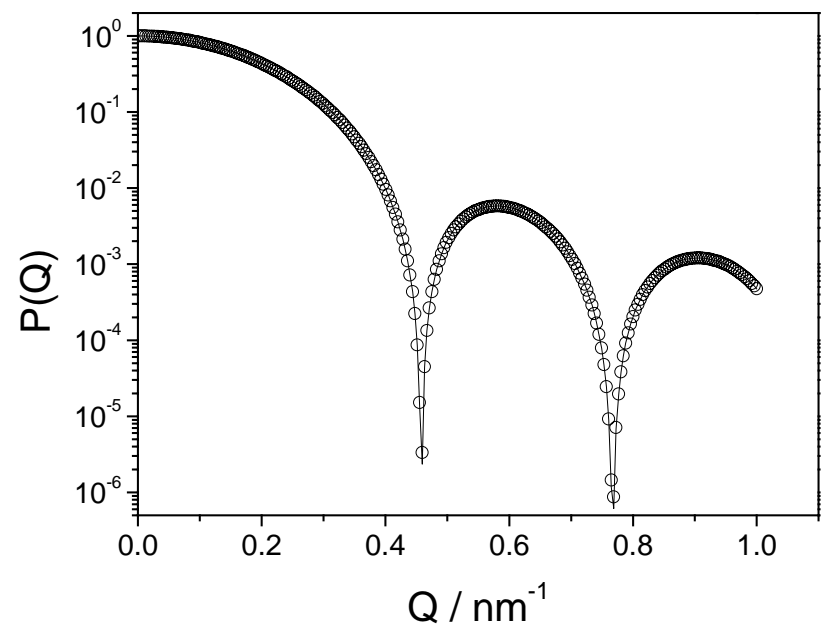


Figure 2 Comparison of the particle scattering factors of a Janus micelle $(O)$ and a the competing core shell structure (-)according to eqs. (1-3) and (5). The structural parameters were set to: $R_{0}=10 \mathrm{~nm}, R_{\mathrm{i}}=5 \mathrm{~nm}, \rho_{\mathrm{A}}=0.956, \rho_{\mathrm{B}}=0.819$ and $\rho_{\mathrm{C}}=0.808$ according to a Janus particle consisting of a polybutadiene core (A) and the two half shells consisting of polystyrene (B) and poly(methymethacrylate) (C). Here data smearing due to limited experimental resolution was not taken into account in the calculations of the form factors.

This can be done rather easily in SANS, because it is very easy to change the scattering contrast, i. e. the excess SLD of certain particle segments by mixing deuterated and proteated solvent. As a consequence the absolute scattering intensity $I(Q)=V_{n}^{2} P(Q)$ at a given scattering vector will change. For particles with segments of different excess scattering length densities the Q-dependence of $\mathrm{P}(\mathrm{Q})$ changes, as well. This is illustrated in Figure 3 were we have collected scattering curves, $I(Q)$ vs $Q$, of Janus micelles and core shell particles at different contrasts.

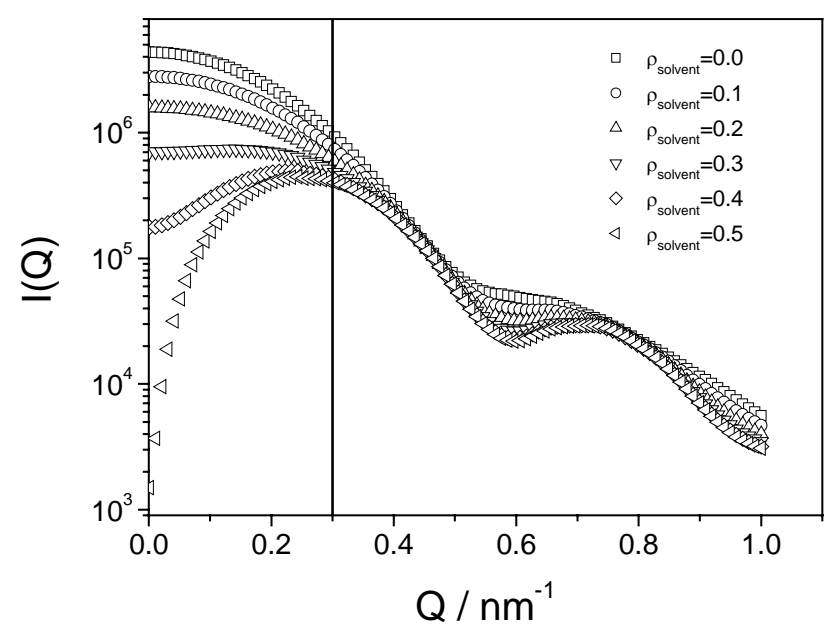




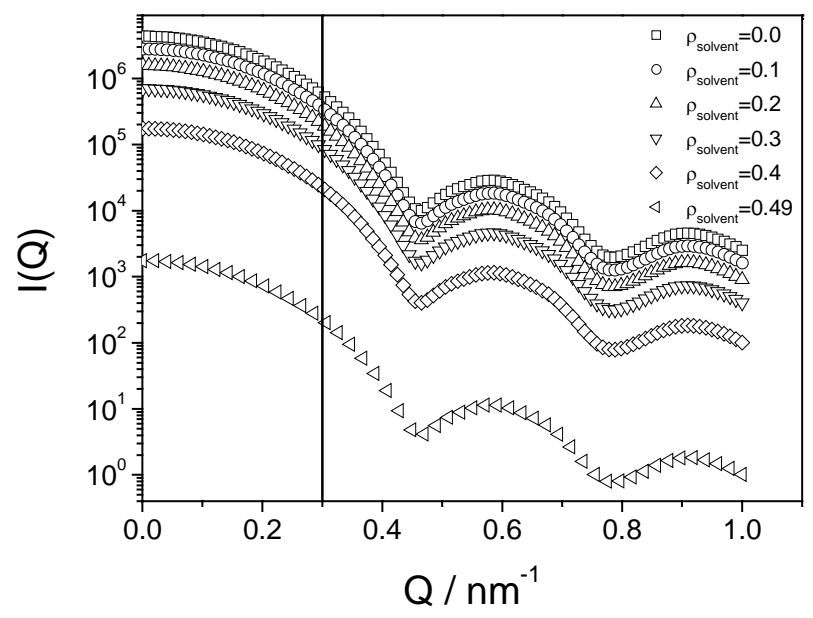

Figure 3 Top: scattered intensity curves for a Janus particle with $R_{0}=10 \mathrm{~nm}, R_{\mathrm{i}}=5 \mathrm{~nm}, \rho_{\mathrm{A}}=0.5$, $\rho_{B}=1.0$ and $\rho_{C}=0.0$. Bottom: scattered intensity curves for the competing 'core shell' structure with $R_{\mathrm{O}}=10 \mathrm{~nm}, R_{\mathrm{i}}=5 \mathrm{~nm}, \rho_{\mathrm{A}}=0.5$ and $\rho_{\mathrm{BC}}=0.5$. The solvent scattering length density varies as indicated in the legend. The vertical lines at $Q_{\mathrm{f}}=0.3 \mathrm{~nm}^{-1}$ represent the cuts along which the absolute intensities at fixed scattering vector were calculated that are plotted in Figure 4.

These curves were calculated for an idealized contrast situation which will be discussed later. However they show that by contrast variation it is well possible to distinguish between the competing structures. Consequently SANS experiments should be performed at a series of solvent SLD and the intensity data should be analyzed in a simultaneous non linear least squares fit of eqs (1-3) to the data. However, the required numerical integrations would cause this strategy to be computationally very time consuming. Even more so if experimental data smearing has to be taken into account. Therefore we suggest a procedure which allows to decide beforehand whether the particles have Janus type or core shell configuration.

In order to distinguish between the two competing structures the most feasible contrast situation is $\rho_{B}=1, \rho_{C}=0$ and $\rho_{A}=0.5$, i.e. the SLD of one half shell matches $\rho_{H-\text { solvent }}^{a b s}$ and that of 
the other matches $\rho_{D \text {-solvent }}^{a b s}$. In this situation the particle scatters as a sphere with constant SLD, if the corona blocks mix completely. That is the angular dependence of $P(Q)$ does not change with the solvent SLD and the absolute intensity scales as $V_{n}^{2}$ at any scattering vector. For such a core shell particle it is possible to match the solvent SLD to the particle. In this case the scattered intensity becomes zero throughout the entire Q-range, because $V_{n}^{2} \rightarrow 0$. For the competing Janus particle the situation is fundamentally different in two respects. (i) $V_{n}^{2}$ can not become zero except for the unphysical case of $R_{0}=0$. (ii) the shape of the particle scattering factor $P(Q)$ varies with the SLD of the solvent. For example, position and relative depth of the form factor minima change with scattering contrast as is shown in Figure 3.

These two differences set the stage for a procedure to distinguish between a Janus micelle and the competing core shell particle. According to point (i) the scattered intensity at a fixed scattering vector from a core shell particle $I_{\mathrm{CS}}\left(Q_{\mathrm{f}}\right)$, runs though a distinct minimum, if the solvent SLD is varied in the range $0 \leq \rho_{\text {solvent }} \leq 1$, as is shown in Figure 4 , where $I_{\mathrm{CS}}\left(Q_{\mathrm{f}}\right)$ vs. $\rho_{\text {solvent }}$ at $Q_{\mathrm{f}} R_{0}=3$ is plotted according to the vertical cuts indicated in Figure 3 . The corresponding curve for the Janus particle is shown as well. Extensive testing of numerous scattering curves showed that a range of $3<Q_{\mathrm{f}} R_{0}<4$ is most suitable for this kind of data analysis. 


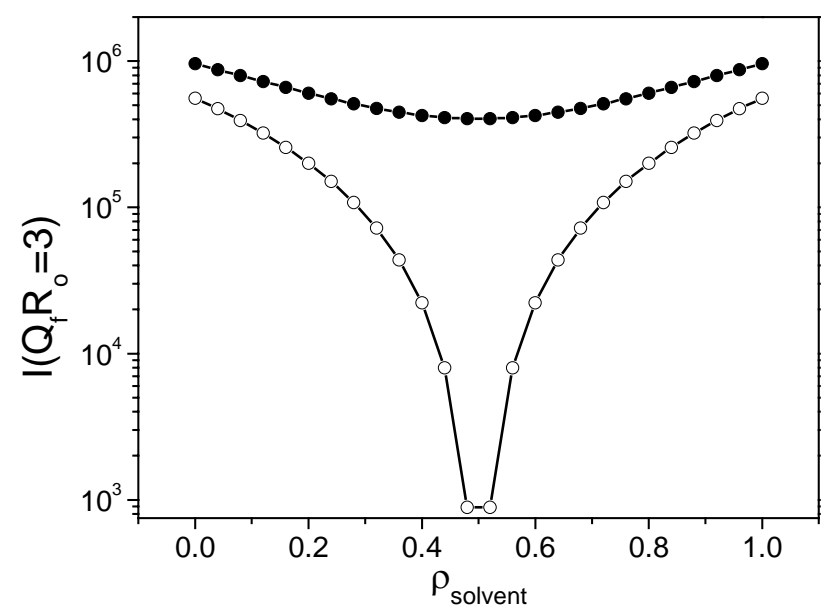

Figure 4 Scattered intensity at fixed $Q_{\mathrm{f}} R_{\mathrm{o}}=3$ for a Janus particle (full circles) with $R_{0}=10 \mathrm{~nm}$, $R_{\mathrm{i}}=5 \mathrm{~nm}, \rho_{\mathrm{A}}=0.5, \rho_{\mathrm{B}}=1.0$ and $\rho_{\mathrm{C}}=0.0$. and for the competing core shell (open circles) structure with $R_{\mathrm{O}}=10 \mathrm{~nm}, R_{\mathrm{i}}=5 \mathrm{~nm}, \rho_{\mathrm{A}}=0.5$ and $\rho_{\mathrm{BC}}=0.5$.

The scattered intensity at $Q_{\mathrm{f}}$ from the Janus particle varies less than a factor of ten while the intensity scattered from the competing core shell structure spans a region of three orders of magnitude as the solvent SLD changes from purely proteated to purely deuterated solvent. This may be regarded as a fingerprint of the core shell structure in contrast to the Janus micelle.

It is probably very difficult, if not impossible to set the SLDs of the respective particle segments by chemical synthesis exactly as we assumed in the above calculation. Therefore we tested whether similarly significant differences are observed for different contrast scenarios and different ratios of the radii. Generally we found that a spread of radii and/or SLD distributions, smears out the dip in the $I\left(Q_{\mathrm{f}} R_{0}=3\right)$ vs. $\rho_{\text {solvent }}$ curves of core shell particles as compared to the curve displayed in Figure 4. Nevertheless, the difference in absolute scattered intensity between the Janus micelle and the core shell particle appears to be a very robust criterion for the distinction between the two competing structures. This is due to the fact that in the case of a core shell 

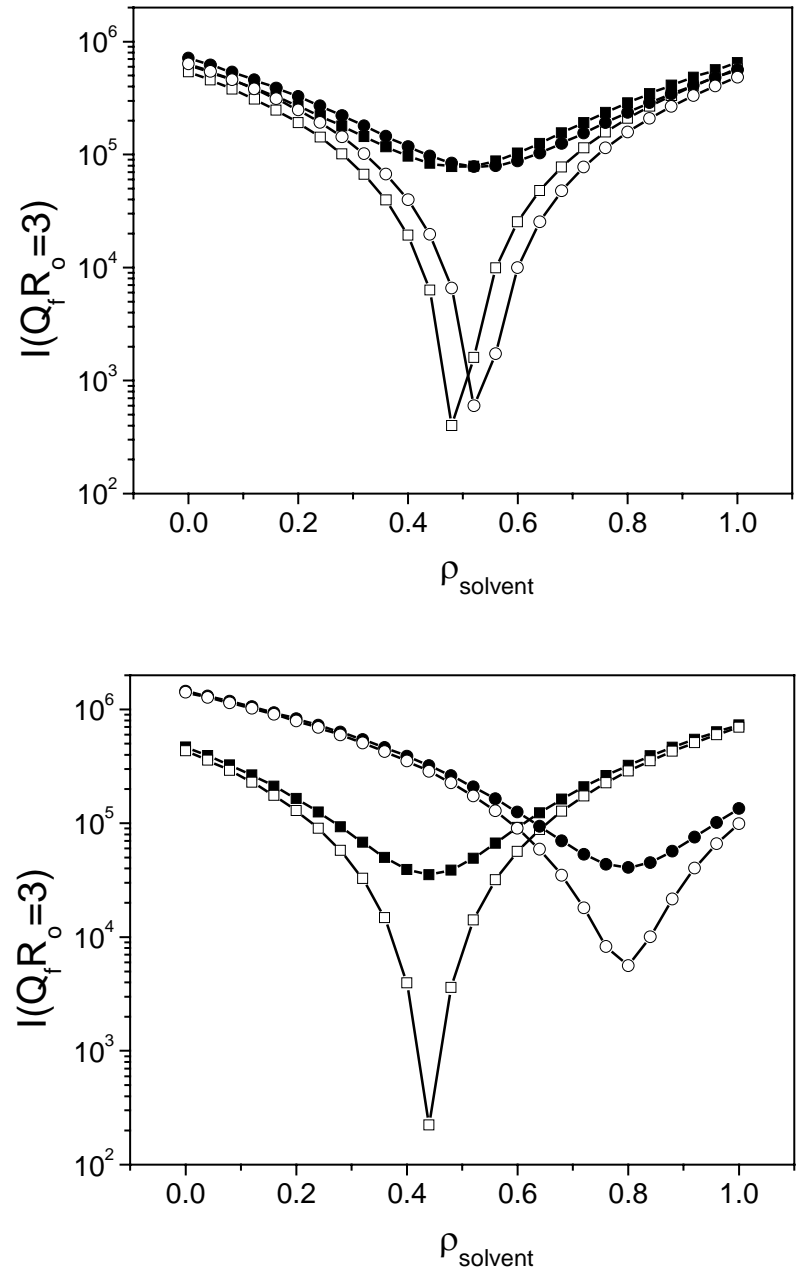

Figure 5 Scattered intensity at fixed $Q_{\mathrm{f}} R_{\mathrm{o}}=3$ for Janus particles (full symbols) and competing core shell structures (open symbols) with $R_{0}=10 \mathrm{~nm}, R_{\mathrm{i}}=3 \mathrm{~nm}$ (top) and $R_{\mathrm{i}}=7 \mathrm{~nm}$ (bottom). The scattering length densities in the corona region were set to $\rho_{B}=0.7, \rho_{C}=0.3$ with $\rho_{B C}=\left(\rho_{C}+\rho_{B}\right) / 2$. For the core we chose $\rho_{\mathrm{A}}=0.4$ (squares) and $\rho_{\mathrm{A}}=1.0$ (circles).

particle there is always a ratio of SLDs which allows for an average matching to the solvent SLD. From the matching condition $V_{\mathrm{n}}=0$ this ratio is easily calculated to be $\rho_{A} / \rho_{B C}=-R_{O}^{3} / R_{i}^{3}$ On the other hand average matching of the particle's SLD to the solvent is not possible in the case of the Janus structure. In this case the matching condition can only be met if $\rho_{B}=\rho_{C}$ which 
again corresponds to a core shell particle. Further examples for the intensity variation with solvent SLD are shown in Figure 5 for four different scenarios.

Generally the curves show only minor changes with the ratio of the radii or the relative SLDs of the segments as long as either $\rho_{B} \leq \rho_{A} \leq \rho_{C}$ or $R_{\mathrm{i}}<R_{0} / 2$. The difference between the competing structures is the least if the core radius approaches the outer particle radius and if the core has the highest SLD of the three particle segments. In this sense the variation of the absolute scattered intensity at an appropriately chosen $Q_{\mathrm{f}}$ with solvent SLD may be regarded as a sound criterion to distinguish between a Janus type particle and the competing core shell structure.

Actually it even holds for systems where the change of SLD between core and corona segments is smeared, as one should expect for real particles. Using the Monte-Carlo scheme described in the section 'Particle Scattering Factor' we have simulated scattering curves for a system where the scattering length density of the core is $\rho_{A}=0.4$ and in the corona segmets the SLDs are $\rho_{B}=0.7$, $\rho_{C}=0.3$ with $\rho_{B C}=\left(\rho_{C}+\rho_{B}\right) / 2$. According to eq. (7) we chose a tanh-function for the continuous transition from the core to the respective corona density. We simulated data for two different ratios of radii, i. e. $R_{\mathrm{i}} / R_{\mathrm{o}}=0.3$ and 0.7 respectively. The width of the transition zone is $d=0.1 R_{\mathrm{i}}$ in both cases. In Figure 6 the scattered intensities at fixed scattering vector are plotted as $I_{\mathrm{CS}}\left(Q_{\mathrm{f}} R_{\mathrm{o}}=3\right)$ vs. $\rho_{\text {solvent }}$ together with the corresponding curve for the Janus micelle. 

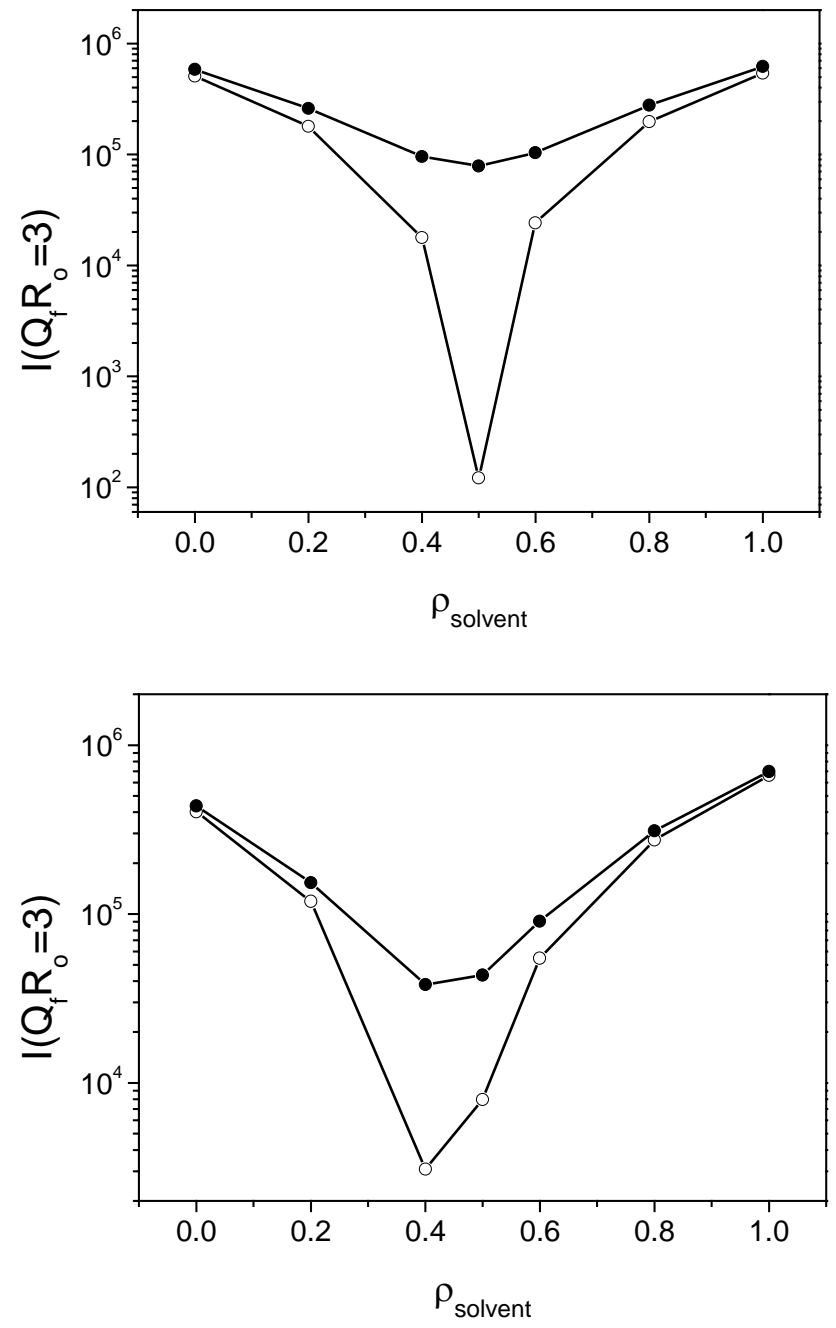

Figure 6 Results of the Monte-Carlo calculations for the scattered intensity at fixed $Q_{\mathrm{f}} R_{0}=3$ for Janus particles (full symbols) and competing core shell structures (open symbols) with $R_{0}=10 \mathrm{~nm}$, $R_{\mathrm{i}}=3 \mathrm{~nm}$ (top) and $R_{\mathrm{i}}=7 \mathrm{~nm}$ (bottom). The scattering length densities in the corona region were set to $\rho_{\mathrm{B}}=0.7, \rho_{\mathrm{C}}=0.3$ with $\rho_{\mathrm{BC}}=\left(\rho_{\mathrm{C}}+\rho_{\mathrm{B}}\right) / 2$. For the core we chose $\rho_{\mathrm{A}}=0.4$. In all cases a continuous density change from the core to the outer particle segment was simulated applying a tanhfunction with a width of $d=0.1 R_{\mathrm{i}}$ according to eq. (7).

It is evident from the top part of Figure 6 that even for particles with a continuous transition from the core SLD to the corona SLD the scattered intensity of the Janus particle varies less than 
a factor of hundred with the solvent SLD at fixed $Q_{\mathrm{f}} R_{\mathrm{o}}=3$ while the scattered intensity from the competing core shell structure has a significant dip with a depth of about three orders of magnitude, if $R_{\mathrm{o}}$ is at least twice as large as $R_{\mathrm{i}}$. However this effect is drastically reduced, if the radius of the core exceeds roughly half of the outer radius, as is to be seen from the bottom part of Figure 6. If $R_{\mathrm{i}} / R_{0}=0.7$ the depth of the minimum in the intensity vs. solvent SLD is only about two orders of magnitude for the core shell structure.

On the basis of this consideration one may conclude that Janus particles are likely to be present, if the variation of the scattered intensity at $Q_{\mathrm{f}} R_{\mathrm{o}}=3$ is less than two orders of magnitude, when the solvent SLD changes from zero to one. In this case the structural parameters of the Janus micelle may be obtained by a simultaneous fit of eqs. (1-3) to experimental scattering curves recorded at a sufficiently high number of different solvent SLD.

CONCLUSION We have shown that the distinction between a Janus-type particle structure and a competing core-shell particle with SANS requires elaborate contrast variation techniques. Based on model calculations with a new particle scattering factor for the Janus structure and simulated scattering data, we suggest a two step procedure to identify and characterize Janus micelles. In the first step the variation of the scattered intensity at a fixed scattering vector $3<Q_{\mathrm{f}} R_{0}<4$ with the solvent SLD should be analyzed. If $I\left(Q_{\mathrm{f}}\right)$ varies less than about two orders of magnitude while $\rho_{\text {solvent }}$ is tuned from zero to one, this may be regarded as strong evidence for the presence of Janus micelles. Only than it is worthwhile, to take the remarkable computational effort of the second step, that is a simultaneous least squares fitting of the I(Q) vs Q data recorded at various contrasts with the model form factor given by eqs. (1-3). As Kaya has already pointed out, it is of large importance that the three segments have a strong tendency to segregation, otherwise the typical features of the scattering curves will be smeared out and eventually make a 
thorough analysis of the data impossible. Further the distinction between the two competing structures is facilitated if, as a rule of thumb, the outer radius is larger than twice the core radius, and if the ratio of the SLDs for the two half shells is as large as possible, typically $\rho_{\mathrm{B}} / \rho_{\mathrm{C}}>2$. The most suitable contrast situation would occur if the core density is $\rho_{A}=\left(\rho_{B}+\rho_{C}\right) / 2$, which is certainly a challenge for the synthesist.

ACKNOWLEDGEMENT We thank G. Nägele for helpful discussions.

APPENDIX The form amplitude of a particle of arbitrary shape is defined as the normalized three dimensional Fourier transform of the particles scattering length density distribution $\rho(\mathbf{r})$. In karthesian coordinates this is

$$
b(\mathbf{Q}, \theta)=\frac{1}{V_{n}} \int \rho(\mathbf{r}) \exp (i \mathbf{Q} \cdot \mathbf{r}) d \mathbf{r}
$$

where the normalization constant in the denominator is the integrated scattering length density $V_{n}=\int \rho(\mathbf{r}) d \mathbf{r}$ and $\theta$ is the angle between the scattering vector $\mathbf{Q}$ and the z-axis. 


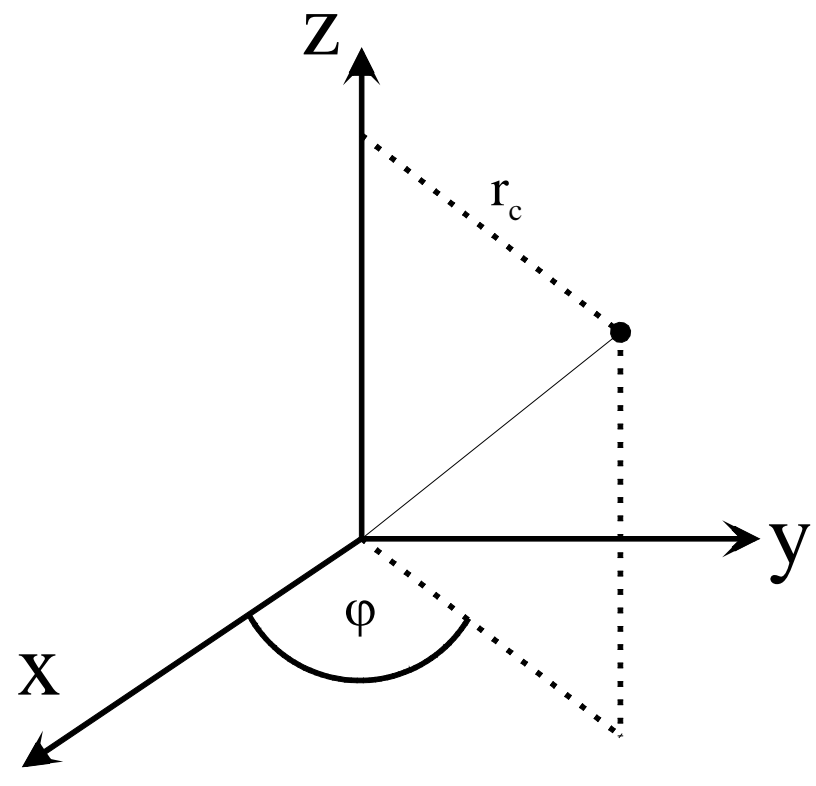

Figure A1 For the definition of the cylindrical coordinates used in the calculations

Although we are considering particles with spherical geometry, it is most convenient to perform the calculations in cylindrical coordinates as they are defined in Fig A1. With these conventions the inner product of scattering vector and positional vector is

$$
\mathbf{Q} \cdot \mathbf{r}=Q\left(r_{c} \sqrt{1-\mu^{2}} \cos \varphi+\mu z\right)
$$

with $\mu=\cos \theta$ and the volume element is $d \mathbf{r}=d r_{C}\left(r_{C} d \varphi\right) d z$.

We now calculate first the form factor of a simple Janus particle, which consists of two half spheres with radius $R_{0}$ each of which has a homogeneous scattering length density

$$
\begin{aligned}
& \rho(\mathbf{r})=\rho_{C} \text { for } 0 \leq \delta<\pi \text { and }|\mathbf{r}| \leq R_{O} \\
& \rho(\mathbf{r})=\rho_{B} \text { for } \pi \leq \delta<2 \pi \text { and }|\mathbf{r}| \leq R_{O}
\end{aligned}
$$

where $\delta$ is the angle between $\mathbf{r}$ and the plane dividing the two half spheres. The form amplitude is then 
$b_{h s}(\mathbf{Q}, \mu)=\frac{1}{V_{n}}\left[\rho_{B} \int_{-R_{o}}^{0} \exp (i Q \mu z) d z+\rho_{C} \int_{0}^{R_{o}} \exp (i Q \mu z) d z\right] \int_{0}^{r_{c} \max } r_{C} d r_{c} \int_{0}^{2 \pi} \exp \left(i Q r_{c} \sqrt{1-\mu^{2}} \cos \varphi\right) d \varphi$

The integral over $\varphi$ is an integral representation of a Bessel function of zero order $J_{0}(x)=\int_{0}^{2 \pi} \exp (i x \cos \alpha) d \alpha / 2 \pi$. For the second integration it has to be taken into account, that the upper limit of $r_{\mathrm{c}}$ is not simply $R_{\mathrm{o}}$ but it depends on $z$, i.e. $r_{c \text {, max }}=\sqrt{R_{O}^{2}-z^{2}}$. If we substitute $Q r_{c} \sqrt{1-\mu^{2}} \equiv x$ and $d x=Q \sqrt{1-\mu^{2}} d r_{c}$, the upper integration limit over $r_{c}$ becomes $u=Q \sqrt{1-\mu^{2}} \sqrt{R_{o}^{2}-z^{2}}$ and the integral may be rewritten as $\sqrt{R_{o}^{2}-z^{2}} J_{1}(u) / Q \sqrt{1-\mu^{2}}$, where we made use of the general property of Bessel functions $\int_{0}^{A} x J_{0}(x) d x=A J_{1}(A)$. Now the form amplitude becomes

$$
b_{h s}(\mathbf{Q}, \mu)=\frac{2 \pi}{V_{n} Q \sqrt{1-\mu^{2}}}\left[\rho_{B} \int_{-R_{o}}^{0} \sqrt{R_{O}^{2}-z^{2}} J_{1}(u) \exp \{i Q \mu z\} d z+\rho_{C} \int_{0}^{R_{o}} \sqrt{R_{o}^{2}-z^{2}} J_{1}(u) \exp \{i Q \mu z\} d z\right]
$$

and the absolute square $\mathrm{b}_{\mathrm{hs}}{ }^{2}(\mathbf{Q}, \mu)=\mathrm{b}(\mathbf{Q}, \mu) \mathrm{b}^{*}(\mathbf{Q}, \mu)$ is

$$
\begin{aligned}
& b_{h s}^{2}(\mathbf{Q}, \mu)=\frac{4 \pi^{2}}{Q^{2} V_{n}^{2}\left(1-\mu^{2}\right)} \\
& \quad\left[\left(\left(\rho_{B}+\rho_{C}\right) \int_{0}^{R_{o}} \cos (Q \mu z) \sqrt{R_{O}^{2}-z^{2}} J_{1}(u) d z\right)^{2}+\left(\left(\rho_{B}-\rho_{C}\right) \int_{0}^{R_{o}} \sin (Q \mu z) \sqrt{R_{O}^{2}-z^{2}} J_{1}(u) d z\right)^{2}\right]
\end{aligned}
$$

The form amplitude of a Janus particle consisting of a core with scattering length density $\rho_{\mathrm{A}}$ and radius $R_{\mathrm{i}}$ covered with two semi-spherical caps of radius $R_{0}$ and scattering length densities $\rho_{\mathrm{B}}$ and $\rho_{\mathrm{C}}$ respectively, can be derived from eq. A4 by replacing a spherical portion in the center of the simple structure by a sphere with $\rho_{\mathrm{A}}$ and $R_{\mathrm{i}}$, which yields 


$$
\begin{aligned}
& b_{J}(\mathbf{Q}, \mu)=\frac{2 \pi}{Q V_{n} \sqrt{1-\mu^{2}}}\left[\rho_{B} \int_{-R_{o}}^{0} \sqrt{R_{O}^{2}-z^{2}} J_{1}\left(u_{O}\right) \exp \{i Q \mu z\} d z\right. \\
& +\left(\rho_{A}-\rho_{B}\right) \int_{-R_{i}}^{0} \sqrt{R_{i}^{2}-z^{2}} J_{1}\left(u_{i}\right) \exp \{i Q \mu z\} d z \\
& +\rho_{C} \int_{0}^{R_{O}} \sqrt{R_{O}^{2}-z^{2}} J_{1}\left(u_{o}\right) \exp \{i Q \mu z\} d z \\
& \left.+\left(\rho_{A}-\rho_{C}\right) \int_{0}^{R_{i}} \sqrt{R_{i}^{2}-z^{2}} J_{1}\left(u_{i}\right) \exp \{i Q \mu z\} d z\right]
\end{aligned}
$$

It is, though rather tedious, straightforward to calculate the absolute square $b_{J}^{2}(\mathbf{Q}, \mu)$ which is given in eq. (3) of the main text.

\section{REFERENCES}

${ }^{1}$ Erhardt, R.; Zhang, M.; Böker, A.; Zettl, H.; Abetz, C.; Frederik, P.; Krausch, G.; Abetz, V.; Müller, A. H. E. J. Am. Chem. Soc. 2003, 125, 3260. Erhardt, R.; Böker, A.; Zettl, H.; Kaya, H.; Pyckhout-Hintzen, W.; Krausch, G.; Abetz, V.; Müller, A. H. E.Macromolecules 2001, 43, 1069 and references given therein.

${ }^{2}$ Bo, Z.; Rabe, J. P.; Schlüter, A. Angew. Chem. 1999, 111, 2540.

${ }^{3}$ Heroguez, V.; Gnanou, Y.; Fontanille, M. Macromolecules 1997, 307, 4791.

${ }^{4}$ Lindlar B.; Boldt M.; Eiden-Assmann S.; Maret, G. Adv. Mater. 2002, 14, 1656.

${ }^{5}$ Koenderink, G. H. "Rotational and translational diffusion in colloidal mixtures” Ph. D. thesis, Universiteit Utrecht, The Netherlands, 2003.

${ }^{6}$ Erdmann, T; Kröger, M.; Hess, S. Phys. Rev. E 2003, 67, 041209 
${ }^{7}$ Kaya, H. Appl. Phys. A 2002, 74, 507.

${ }^{8}$ Pedersen, J. S. Adv. Coll. Interf. Sci. 1997, 70, 171.

${ }^{9}$ Barker, J. G. Pedersen, J. S. J. Appl. Cryst. 1995, 28, 105. 